\title{
Impact du chancre hypoxylonien sur le tremble de 2 unités de gestion du Québec
}

\author{
par
}

\author{
Louis Archambault ${ }^{1}$
}

\section{Résumé}

Un inventaire pour évaluer l'impact du chancre hypoxylonien (Hypoxylon mammatum (Wahl.)) chez le peuplier faux-tremble (Populus tremuloides Michx.) a été réalisé dans les unité de gestion de forêt publique de Portneuf et Grand-Portage de la province de Québec. Dans Portneuf, 78 peuplements forestiers ont été échantillonnés et 2340 tiges ont été analysées tandis que dans Grand-Portage 82 peuplements (2460 tiges) ont été visités. Dans l'unité de Portneuf, $2.6 \%$ des tiges (2.0\% du volume) étaient affectées ou mortes par la maladie comparativement à $4.1 \%$ des tiges $(2.8 \%$ du volume) dans Grand-Portage. D'autres analyses portent sur l'incidence de la maladie en fonction des caractéristiques dendrométriques des peuplements.

Quoique relativement faibles, les pertes annuelles, qui ne représentent que le sixième de cette évaluation, s'additionnent tout au long de la vie des peuplements. De plus cet impact pourrait devenir plus significatif dans l'éventualité d'une plus grande utilisation du peuplier faux-tremble.

\section{Abstract}

A survey was conducted in the management units of Portneuf and Grand-Portage, Québec to determine the impact of Hypoxylon canker (Hypoxylon mammatum (Wahl.)) on aspen (Populus tremuloides Michx.). In 78 forest stands (2340 stems) of Portneuf, sampling revealed $2.6 \%$ of stems $(2.0 \%$ of volume) affected or dead. In the 82 Grand-Portage stands (2460 stems) $4.1 \%$ of stems $(2.8 \%$ of volume) were affected or dead. Other analyses were carried out on the frequency of the disease in relation to stand characteristics.

Although annual losses which represent only a sixth of this evaluation are relatively low, we must emphasize that they are cumulative over the whole life of the stands. Moreover, the importance of the impact could increase if aspen was widely used.

\section{Introduction}

Au Québec, le peuplier faux-tremble (Populus tremuloides Michx.) est une esssence forestière dont l'importance économique pourrait s'accroître au cours des prochaines années. En volume, le genre Populus représentente environ $5.6 \%$ des stocks ligneux de cette province (Barrette et Langevin 1979 Boivin et Lafrance 1977) et le peuplier fauxtremble est de loin la principale espèce du genre. Le chancre hypoxylonien causé par Hypoxylon mammatum (Wahl.) est une maladie très fréquente du tremble. Sa distribution presque universelle à travers le Canada, le nord-est et le

${ }^{1}$ Centre de Recherches forestières des Laurentides, Service canadien des forêts, 1080. route du Vallon, Sainte-Foy, Québec, G1V 4C7.

${ }^{2}$ Anderson, R.L. Ph.D. thesis University of Minnesota. centre-nord des Etats-Unis (Lavallée 1978) entraîne chez cette essence forestière des pertes appréciables de matière ligneuse.

Des études ont démontré que dans certains peuplements forestiers détériorés le chancre peut affecter de 50 à $70 \%$ des arbres parmi lesquels 15 à $25 \%$ sont déjà morts ou brisés (Lavallée 1978). D'autre part, Anderson (1964) affirme que pour les états du Wisconsin, du Minnesota et du Michigan les pertes annuefles attribuées au chancre se chiffrent à $1 \%$ du volume total de tremble et à $30 \%$ de son accroissement annuel moyen.

Malgré ces quelques données on constate qu'au Québec nos connaissances de l'impact du chancre en terme de perte de matière ligneuse sont très fragmentaires. Afin de mieux évaluer l'importance des problèmes engendrés par ce pathogène, nous avons réalisé l'échantillonnage de 2 territoires particuliers du Québec soient les unités de gestion de Portneuf et du Grand-Portage.

\section{Méthode}

L'inventaire effectué lors de l'automne 1980 et de l'été 1981 comportait l'étude de deux unités de gestion de forêt publique, tel que défini par le ministère de l'énergie et des Ressources du gouvernement du Québec, à savoir les unités de Portneuf et du Grand Portage. Ces dernières font toutes deux partie de la région forestière des Grands Lacs et du St-Laurent (Rowe 1972). L'unité de Portneuf située à environ $20 \mathrm{~km}$ au sud-ouest de la ville de Québec a été inventoriée dans sa partie sud seulement soit un territoire d'environ $3000 \mathrm{~km}^{2}$ limité au nord par le petit lac Batiscan. Quant à l'unité de Grand-Portage qui est située approximativement à $200 \mathrm{~km}$ au nord-est de la ville de Québec l'ensemble de son territoire (environ $9000 \mathrm{~km}^{2}$ ) a été inventorié (Figure 1).

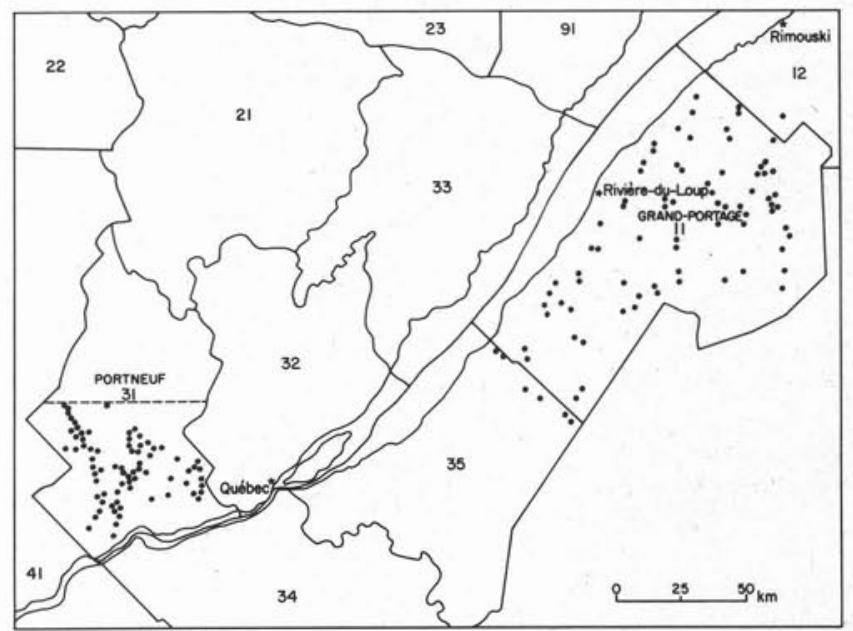

Figure 1. Localisation des unités d'aménagement inventoriées a) Sud de l'unité de Portneuf (31)

b) Unité du Grand-Portage (11)

c) peuplement échantilloné 
Dans les 2 unités, les terrains publics et privés ont été considérés. Les peuplements forestiers susceptibles de contenir du peuplier faux-tremble selon les normes du ministère de l'Energie et des Ressources (Québec 1975) ont été choisis au hasard sur les cartes forestières des unités. Pour être choisi le peuplement devait être facilement accessible par route.

Les peuplements ont été distribués sur l'ensemble des unités de façon à obtenir une couverture totale de ces dernières. Trois parcelles-échantillons étaient établies dans les endroits les plus représentatifs des caractères dendrométriques des peuplements sélectionnés. La parcelle-échantillon consistait en bande linéaire d'environ trois mètres de largeur dans laquelle les 10 premiers peupliers faux-tremble d'au moins $4 \mathrm{~cm}$ de diamètre étaient étudiés.

Le diamètre à hauteur de poitrine (D.H.P.) de toutes ces tiges a été mesuré et les caractéristiques suivantes ont été notées sur les arbres atteints du chancre: hauteur, étage, nombre et hauteur des chancres sur la tige. Pour qu'un tremble mort soit considéré, nous devions avoir la certitude que la cause principale de sa mort ait été le chancre hypoxylonien, c'est-à-dire que les symptômes de la maladie soient encore visibles sur l'arbré.

Ainsi dans Portneuf 78 peuplements (2340 tiges) ont été choisis tandis que dans Grand-Portage ce nombre a été de 82 (2460 tiges).

\section{Résultats}

Le pourcentage des tiges affectées ou mortes pour l'ensemble des parcelles fut de $2.6 \%$ (2.0\% du volume marchand) dans Portneuf et de $4.1 \%$ (2.8\% du volume mar- chand) dans Grand-Portage pour un total de $2.5 \%$ du volume total échantillonné (Tableau 1).

Dans Portneuf $25(32.0 \%)$ des peuplements visités étaient affectés à divers degrés avec un pourcentage moyen d'infection de $8.1 \%$. Pour Grand-Portage ces résultats sont respectivement $48(58.4 \%$ ) et $7.1 \%$ (Tableau 2). Le pourcentage d'infection des peuplements a varié entre 0 et $26.7 \%$ pour les 2 unités.

L'analyse de l'incidence de la maladie en fonction des caractéristiques dendrométriques des peuplements, révèle qu'en considérant les 2 unités réunies les jeunes peuplements feuillus de faible densité sont les plus susceptibles à la maladie, tandis que les peuplements mélangés mûrs résistent le mieux à l'envahissement du chancre. L'impact de la maladie est environ dix fois supérieur chez les jeunes peuplements feuillus de faible densité que chez les peuplements mélangés mûrs. Chez les peuplements feuillus l'intensité de l'attaque diminue lorsque la densité du peuplement augmente tandis que chez les peuplements mélangés l'impact du chancre diminue lorsqu'ils sont plus âgés (Tableau 3).

D'autre part le taux d'infection des peuplements dont la proportion de tremble dépasse $75 \%$ est deux fois plus grand que chez les peuplements ayant moins de $25 \%$ de leur volume en tremble (Tableau 4). Enfin, il semble que de façon générale les peuplements feuillus soient plus enclins que les peuplements mélangés à subir les inconvénients de la maladie.

Dans Portneuf, le diamètre à hauteur de poitrine (DHP) des tiges attaquées a varié entre 6 et $24 \mathrm{~cm}$ et $97.0 \%$ de ces dernières avaient un DHP de $20 \mathrm{~cm}$ et moins. Dans Grand-Portage ces DHP ont oscillé entre 4 et $34 \mathrm{~cm}$ avec

Tableau 1. Résultats comparatifs de l'inventaire du chancre hypoxylonien.

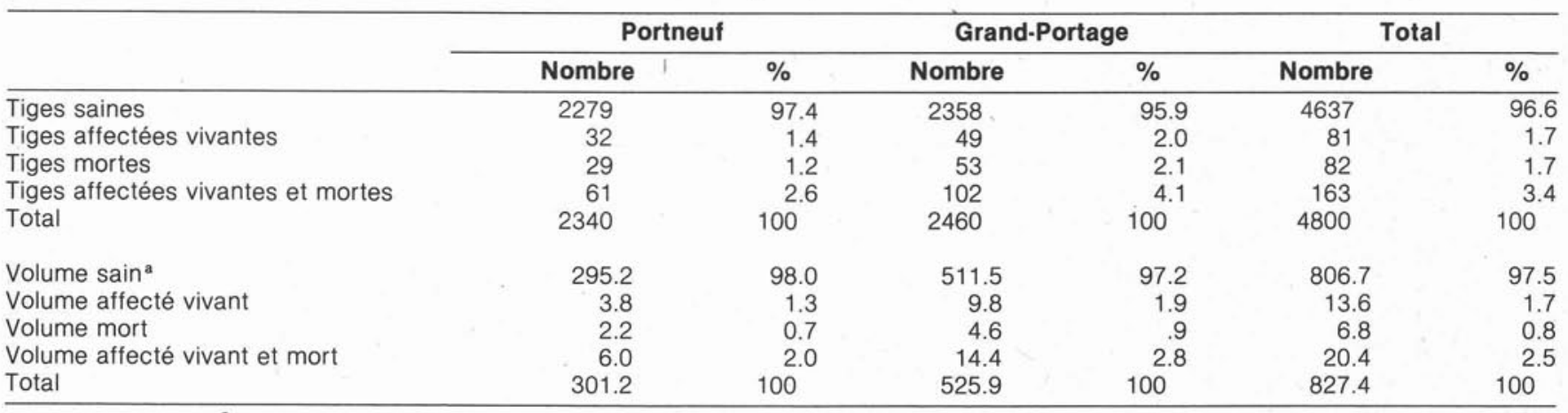

: volume marchand en $\mathrm{m}^{3}$

Tableau 2. Susceptibilité des peuplements en fonction de leurs caractéristiques dendrométriques.

\begin{tabular}{|c|c|c|c|c|c|c|c|c|}
\hline \multirow{2}{*}{\multicolumn{3}{|c|}{ Type de peuplement }} & \multicolumn{2}{|c|}{ Portneuf } & \multicolumn{2}{|c|}{ Grand-Portage } & \multicolumn{2}{|c|}{ Total } \\
\hline & & & $\begin{array}{c}\text { Nombre de } \\
\text { peuplements } \\
\text { étudiés }\end{array}$ & $\begin{array}{c}\% \text { des } \\
\text { peuplements } \\
\text { affectés }\end{array}$ & $\begin{array}{c}\text { Nombre de } \\
\text { peuplements } \\
\text { étudiés }\end{array}$ & $\begin{array}{c}\% \text { des } \\
\text { peuplements } \\
\text { affectés }\end{array}$ & $\begin{array}{c}\text { Nombre de } \\
\text { peuplements } \\
\text { étudiés }\end{array}$ & $\begin{array}{c}\% \text { des } \\
\text { peuplements } \\
\text { affectés }\end{array}$ \\
\hline $\mathbf{R}$ & $a \cdot b$ & M & 0 & 0 & 1 & 100 & 1 & 100 \\
\hline \multicolumn{3}{|c|}{ Total } & 78 & 32.0 & 82 & 58.4 & 160 & 45.6 \\
\hline
\end{tabular}

$1_{\mathrm{F}}=$ Feuillu, $\mathrm{M}=$ Mélangé, $\mathrm{R}=$ Résineux

${ }^{2}$ Densité du peuplement. $a-b=$ forte densité $(>60 \%) c-d=$ faible densité $(\$ 60 \%)$

3 Age du peuplement. $J=$ jeune $(<40$ ans) $M=$ mûr ( $\geqslant 40$ ans). 
Tableau 3. Pourcentage moyen d'infection des peuplements en fonction de leurs caractéristiques dendrométriques.

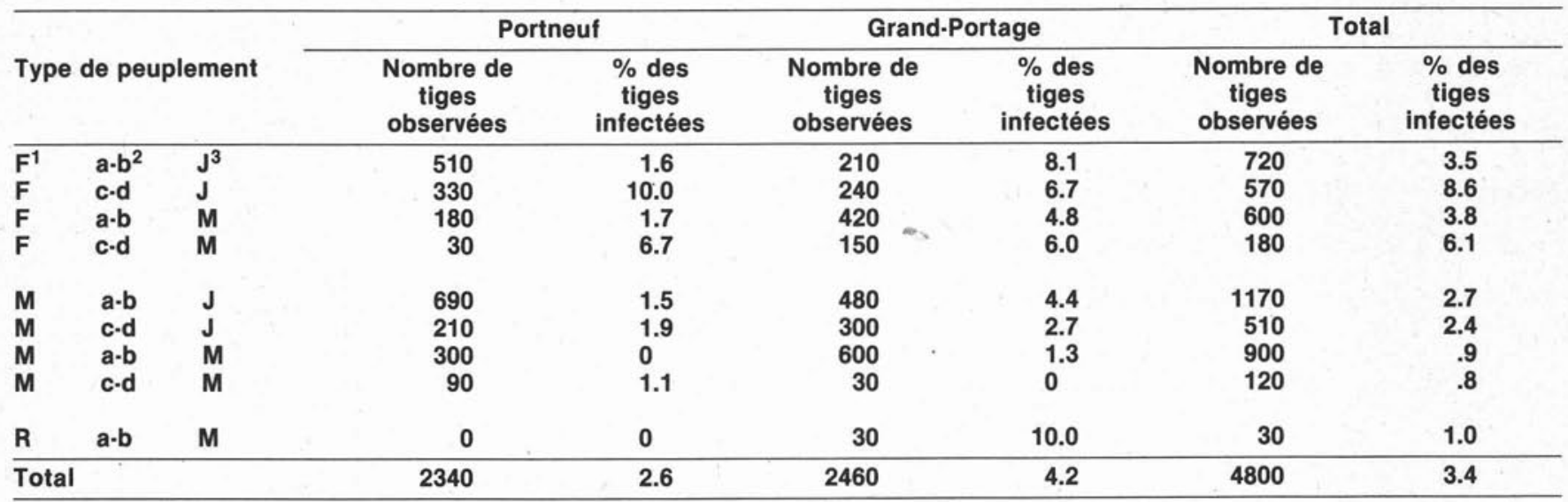

${ }^{1} \mathrm{~F}=$ Feuillu, $\mathrm{M}=$ Mélangé, $\mathrm{R}=$ Résineux

${ }^{2}$ Densité du peuplement. $a-b=$ forte densité $(>60 \%) c-d=$ faible density $(\leqslant 60 \%)$

${ }^{3}$ Age du peuplement. $\mathrm{J}=$ jeune $(<40$ ans) $\mathrm{M}=$ mûr $(\geqslant 40$ ans).

Tableau 4. Pourcentage moyen d'infection des peuplements en fonction de leur proportion de tremble (en volume).

\begin{tabular}{lcccccc}
\hline & \multicolumn{2}{c}{ Portneuf } & \multicolumn{2}{c}{ Grand-Portage } & \multicolumn{2}{c}{ Total } \\
\cline { 2 - 7 } $\begin{array}{l}\text { Proportion de } \\
\text { tremble en } \\
\text { volume (\%) }\end{array}$ & $\begin{array}{c}\text { Nombre de } \\
\text { peuplements } \\
\text { étudiés }\end{array}$ & $\begin{array}{c}\text { Pourcentage } \\
\text { des tiges } \\
\text { infectées }\end{array}$ & $\begin{array}{c}\text { Nombre de } \\
\text { peuplements } \\
\text { étudiés }\end{array}$ & $\begin{array}{c}\text { Pourcentage } \\
\text { des tiges } \\
\text { infectées }\end{array}$ & $\begin{array}{c}\text { Nombre de } \\
\text { peuplements } \\
\text { étudiés }\end{array}$ & $\begin{array}{c}\text { Pourcentage } \\
\text { des tiges } \\
\text { infectées }\end{array}$ \\
\hline $0-25$ & 44 & 2.0 & 16 & 4.6 & 60 & 2.7 \\
$25-50$ & 11 & 3.6 & 25 & 3.3 & 3.4 & 3.6 \\
$50-75$ & 15 & 2.4 & 31 & 4.1 & 18 \\
$75-100$ & 8 & 5.0 & 10 & 5.7 & 5.4 \\
Total & 78 & 2.6 & 82 & 4.1 & 160 & 3.4 \\
\hline
\end{tabular}

$84.3 \%$ des tiges attaquées ayant un D.H.P. de $20 \mathrm{~cm}$ et moins. Au total l'échantillonage de cette catégorie de tiges (20 cm et moins) représente respectivement $85.7 \%$ et $71.1 \%$ du nombre total de tiges analysées dans Portneuf et Grand-Portage. Pour les 2 unités réunies le volume marchand total attaqué ou mort représente $2.5 \%$ du volume total échantillonné, les tiges attaquées de $20 \mathrm{~cm}$ ou moins de diamètre comptant pour $1.0 \%$ du volume total échantillonné tandis que celles de plus de $20 \mathrm{~cm}$ représentent $1.5 \%$ de ce volume.

Enfin signalons que les chancres ont été observés sur des tiges de toutes les classes d'étage et de hauteur.

\section{Discussion}

Le plus haut taux d'infection observé dans les 160 peuplements échantillonnés a été de $26.7 \%$ ce qui est inférieur aux observations de Lavallée (1978). Comme Anderson and Martin (1981) nous avons constaté que les peuplements dont la proportion de tremble est plus importante sont les plus touchés par la maladie. Certains auteurs (Anderson 1957 and Anderson 1968, Anderson and Martin 1981) ont noté que l'incidence de la maladie augmentait lorsque la densité du peuplement diminuait. Nos observations confirment celles de ces auteurs en ce qui concerne les peuplements feuillus, par contre chez les peuplements mélangés la densité ne semble pas reliée à l'impact du chancre. Gruenhagen (1945) et Anderson² (1952) soulignent que le chancre est plus fréquent chez les jeunes peuplements. Nos résultats concordent avec cette observation pour les peuplements mélangés tandis que chez les peuplements feuillus l'âge ne semble pas être en relation avec l'incidence de la maladie. Comme Anderson (1956) nous avons constaté que les tiges vigoureuses et dominantes peuvent être attaquées au même titre que celles qui sont faibles ou opprimées.

Un aspect important de cette étude est qu'à l'échelle des 2 unités d'aménagement environ $2.5 \%$ du volume marchand total est affecté ou mort. De plus, $60.1 \%$ des pertes en volume se retrouvent chez les tiges de plus de $20 \mathrm{~cm}$ de D.H.P. donc des tiges ayant un potential commercial. II importe cependant de souligner qu'en moyenne, les tiges mettent 6 ans à mourrir (Anderson and Martin 1981) diminuant de beaucoup les pertes annuelles dues au chancre.

Bien que cet inventaire ne porte que sur un territoire restreint du Québec ces résultats sont relativement représentatifs de la situation du chancre hypoxylonien au Québec. En effet, un relevé spécial réalisé en 1981 par la section du Relevé des insectes et maladies des arbres du Centre de Recherches forestières des Laurentides (Benoit et $\mathrm{al}$. 1982) révèle que $5.0 \%$ des tiges $(4.0 \%$ du volume marchand) de tremble sont atteintes par la maladie. Ces résultats proviennent de 336 parcelles-échantillons réparties sur l'ensemble du territoire québécois. Ces données quoique supérieures aux nôtres sont du même ordre de grandeur.

\section{Conclusion}

Les résultats de cet inventaire sont ponctuels et nous donnent une image de la forêt à un moment particulier. Cependant, la situation est dynamique et les pertes annuelles s'additionnent tout au long de la vie du peuplement. Actuellement la demande pour le peuplier faux-tremble est relativement peu importante, mais cette situation pourrait évoluer dans le futur compte tenu de la demande sans cesse croissante de l'ensemble des produits du bois. Enfin compte tenu des coûts occasionnés par l'éloignement de la 
matière ligneuse des usines de transformation les forestiers devront pratiquer un aménagement intensif des meilleurs sites et devront tenir compte de tous les facteurs pouvant avoir un impact sur la production des terrains forestiers mis à leur disposition.

\section{Références}

Anderson, R.L. 1952. Factors influencing the incidence of Hypoxylon canker of aspen. Phytopathology 42: 463.

Anderson, R.L. 1956. Hypoxylon canker of aspen. USDA Forest Service, Forest pest leaflet $6.7 p$.

Anderson, R.L. 1964. Hypoxylon canker of aspen. Phytopathology 54(3): 253-257

Anderson, G.W. and R.L. Anderson. 1968. Relationship between density of quaking aspen and incidence of Hypoxylon canker. For Sci. 14: 107-112.
Anderson, G.W. and M.P. Martin. 1981. Factors related to incidence of hypoxylon canker in aspen and survival of cankered trees. For. Sci. 27: 461-476.

Benoit, P., G. Laflamme, G. Bonneau et R. Picher. 1982. Insectes et maladies des arbres 1981. Supplément ForêtConserv. 48 (9).

Barrette, Y. et D.P. Langevin. 1979. Ressources et activités en forêt privée. Qué. Minist. Terres For. Pub. TFI 4000-2. 144 p.

Boivin, J.L. et R. Lafrance. 1977. Les feuillus du Québec 1. La ressource. Qué. Minist. Terres For. Qué. 201 P.

Gruenhagen, R.H. 1945. Hypoxylon pruinatun and its pathogenesis on poplar. Phytopathology 35: 72-89.

Lavallée, A. 1978. Le chancre hypoxylonien du peuplier. Serv. Can. For. Feuillet d'infor. C.R.F.L. $n^{\circ} 216 \mathrm{p}$.

Québec. 1975. Normes d'inventaire forestier 1975. Qué. Minist. Terres For. Pub. TFC-3209-11a.

Rowe, J.S. 1972. Les régions forestières du Canada. For. Pub. 1300F. 172 p.

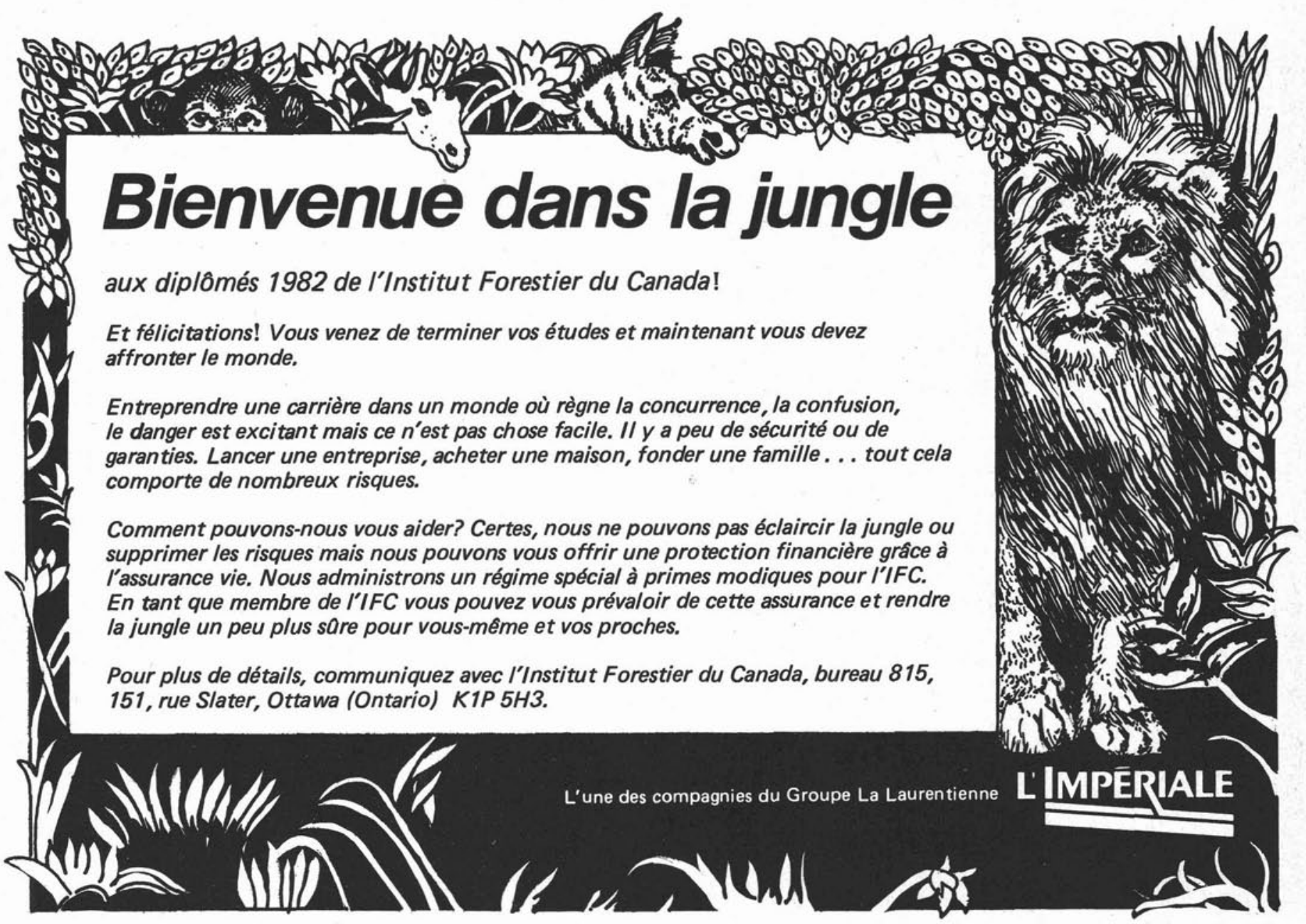

